\title{
Diagnostic assessments of spirometry and medical history data by respiratory specialists supporting primary care: are they reliable?
}

\section{*Annelies EM Lucas ${ }^{a}$, Frank JWM Smeenk ${ }^{b}$, Ben EEM van den Borne ${ }^{b}$, Ivo JM Smeelec, Onno CP van Schayck ${ }^{a}$}

\footnotetext{
a Research Institute Caphri, Department of General Practice, Maastricht University, Maastricht, The Netherlands

${ }^{b}$ Department of Pulmonology, Catharina Hospital, Eindhoven, The Netherlands

C COPD \& Asthma Primary Care Group (CAHAG), Utrecht, The Netherlands
}

Received 8th January 2008; resubmitted 9th March 2008; revised 18th July 2008; accepted 25th September 2008; online 12th January 2009

\begin{abstract}
Aim: To determine the intra- and inter-observer reliability of respiratory specialists' diagnostic assessments of spirometry and written medical history data obtained from primary care.

Method: Five respiratory specialists assessed spirometry data and the history of 156 patients randomly selected from referrals to an asthma/COPD-service. The inter-observer reliability was evaluated. After six months, all specialists repeated the assessments and the intraobserver reliability was evaluated.

Results: The diagnostic assessments for all patients had reasonable intra- and inter-observer reliability, resulting in a Cohen's kappa ( $\kappa$ ) of 0.67 and 0.66 respectively. The intra-observer reliability for assessing the need for additional diagnostic examinations had an average $\kappa 0.56$ for new patients and an average $\kappa 0.39$ for follow-up examinations. The assessments of clinical stability in follow-up patients - on which therapeutic advice was based - were inconsistent.

Conclusion: GPs who are reluctant to perform or interpret spirometry themselves may be supported diagnostically by respiratory specialists in an asthma/COPD-service. The reliability of this advice varies. More appropriate criteria for assessing clinical stability in patients with asthma and COPD are necessary to improve the reliability of the therapeutic advice.

(C) 2009 General Practice Airways Group. All rights reserved.

AEM Lucas, et al. Prim Care Resp J 2009; 18(3): 177-184

doi:10.3132/pcrj.2009.00002
\end{abstract}

Keywords spirometry, primary care, asthma, COPD, diagnosis

\section{See linked editorial by Jenkins on pg 128}

\section{Introduction}

In primary care, practical thresholds hinder the implementation of spirometry, ${ }^{1,2}$ and many general practitioners (GPs) encounter problems in interpreting spirometry data. ${ }^{3}$ Therefore, in several countries facilities have been developed to support GPs in assessing the diagnosis of asthma or COPD in patients with respiratory complaints. These facilities provide spirometry testing assessed by speciallytrained $\mathrm{GPs}^{4}$ or respiratory specialists. ${ }^{5}$ Some services only interpret the spirometry results, ${ }^{6}$ whereas others include $x$-ray and $\mathrm{O}_{2}$-saturation ${ }^{7}$ assessments or use medical history data; ${ }^{8}$ furthermore, the respiratory specialists can collect data by actually seeing the patient ${ }^{9}$ or by assessing paper-based information only. ${ }^{10}$ In addition, computerised data assessment is used on a wide scale. ${ }^{11}$

The reliability and validity of these assessment procedures may be questioned, especially when a patient is not actually seen by the assessor. Therefore, we examined the validity and reliability of diagnostic assessments based on spirometry results and written medical histories. In a previous study we found that these assessments had good validity: there were high similarities between the assessments of a pulmonologist

\footnotetext{
* Corresponding author: Mrs Annelies EM Lucas, Research Institute Caphri, Department of General Practice (HAG), University of Maastricht, PO Box 616, 6200 MD Maastricht, The Netherlands. Tel: +31 (0)43 3882149 Fax: +31 (0)43 3619344 E-mail: Annelies.Lucas@Hag.unimaas.nl
} 
based on written information and the assessments of the same pulmonologist based on "live" contact with the patient. ${ }^{12}$ In this paper, we examine the reliability of diagnostic assessments by different respiratory specialists who used spirometry data and written patient information. Our specific questions were:

What are the intra- and inter-observer reliability of

1. the actual diagnoses given to the patient?

2. the advice for additional diagnostic examinations in case of an unclear initial diagnosis ?

3. the assessment of the clinical stability of the patient and the subsequent advice for adjustments in the treatment regime?

\section{Method}

\section{Asthma/COPD-service}

The study was performed at a Primary Care Diagnostic Centre in Eindhoven in the Netherlands. This centre has developed an asthma/COPD-service (AC-service). About 200 GPs refer their patients with respiratory problems to the centre for diagnosis and monitoring, and in 2007 approximately 6000 referrals were made. When referred, a patient fills in a structured form with questions about their medical history, smoking behaviour, fitness, complaints (symptoms), medical treatment and compliance. This medical history form is taken to the ACservice where well trained lung function assistants perform spirometry including reversibility testing.

The medical history form and the results of spirometry are kept together and sent to co-operating respiratory specialists from local hospitals. They perform diagnostic assessments according to an assessment protocol that includes the following criteria:

- does the quality of the performed spirometry comply with ATS criteria ${ }^{13}$ ?

- does the flow volume curve show obstruction (FEV 1 /FVC $<0.7)$ and is this obstruction reversible after inhaling bronchodilator medication? (According to the Dutch standard the difference between $\mathrm{FEV}_{1}$ before and after bronchodilation should be $>9 \%$ of the $\mathrm{FEV}_{1} \%$ predicted). ${ }^{14}$

- what is the conclusion based upon the patient's lung function curves?

1. No obstruction = no asthma/COPD;

2. No obstruction but decline in $\mathrm{FEV}_{1}$ and in $\mathrm{FVC}=$ restriction

3. Obstruction is reversible to normal lung function = asthma

4. Irreversible obstruction $=$ COPD

5. Obstruction is reversible but not to normal lung function = both asthma and COPD

- (how) does the patient's medical history (allergy, family history, smoking behaviour, complaints) influence the final diagnosis - for example, in a case of normal lung function which does not necessarily exclude asthma?

- (how) does the patient's medical history (physical shape, complaints, exacerbations, use of medication etc.) influence the assessment of the stability of the disease and the patient's condition?

Following this protocol the respiratory specialist assesses a diagnosis and gives therapeutic advice. In case of a discrepancy between the symptoms and the spirometry results, advice is given to the GP to conduct additional examinations in order to determine the final diagnosis. Referral to a pulmonologist is recommended according to the Dutch guidelines. ${ }^{15}$ All assessment items are marked on a structured assessment form. A complete report (Figure 1) is then made by the AC-service and sent to the patient's GP.

\section{New patients and follow-up patients}

For newly-referred patients (New Patients) the focus is on being diagnosed, or (if necessary) on obtaining advice for further diagnostic examinations. When a diagnosis of "asthma" or "COPD" is assessed, patients can be included in a monitoring program that invites them to visit the $\mathrm{AC}$-service every year. For these "follow-up" patients, the focus is on assessing instability and progression of the disease and on providing therapeutic advice. For both new and follow-up patients the complete assessment procedure is performed: new patients also get therapeutic advice, when applicable, and follow-up patients are also checked for their diagnosis.

\section{Patient data}

During six months (January - July 2004) data records of 156 patients who were referred to the asthma/COPD-service were randomly selected (the first 7-8 patients of each week). Five respiratory specialists, who did not consult each other, assessed the spirometry results and medical history forms of the selected patients according to the protocol. Six months later all five assessors examined copies of the same patient data for a second time. These copies were mixed in with the regular daily assessments to avoid recall bias.

\section{Intra- and inter-observer comparison method}

We first analysed the intra-observer reliability for all five observers by comparing the assessment forms of two assessments by each assessor of all patients. Only when the intra-observer reliability was reasonable for all observers ( $\kappa>0.5$ ) was the inter-observer reliability then assessed.

Specific items for analysing the intra- and inter-observer agreement

To answer our first question about the reliability of the assessed diagnoses the scores of all diagnostic options were compared. These diagnostic options were:

1. "Normal, no asthma or COPD'

2. "Asthma"

3. "Asthma with persisting obstruction" (i.e. mixed Asthma and (OPD)

4. "COPD"

5. "Restriction, no asthma or COPD" 
Respiratory specialists diagnostic support: reliable?

Figure 1. Example of a report of the diagnostic assessment by a pulmonologist based on written information as sent to the general practitioner by the Asthma/COPD-service.

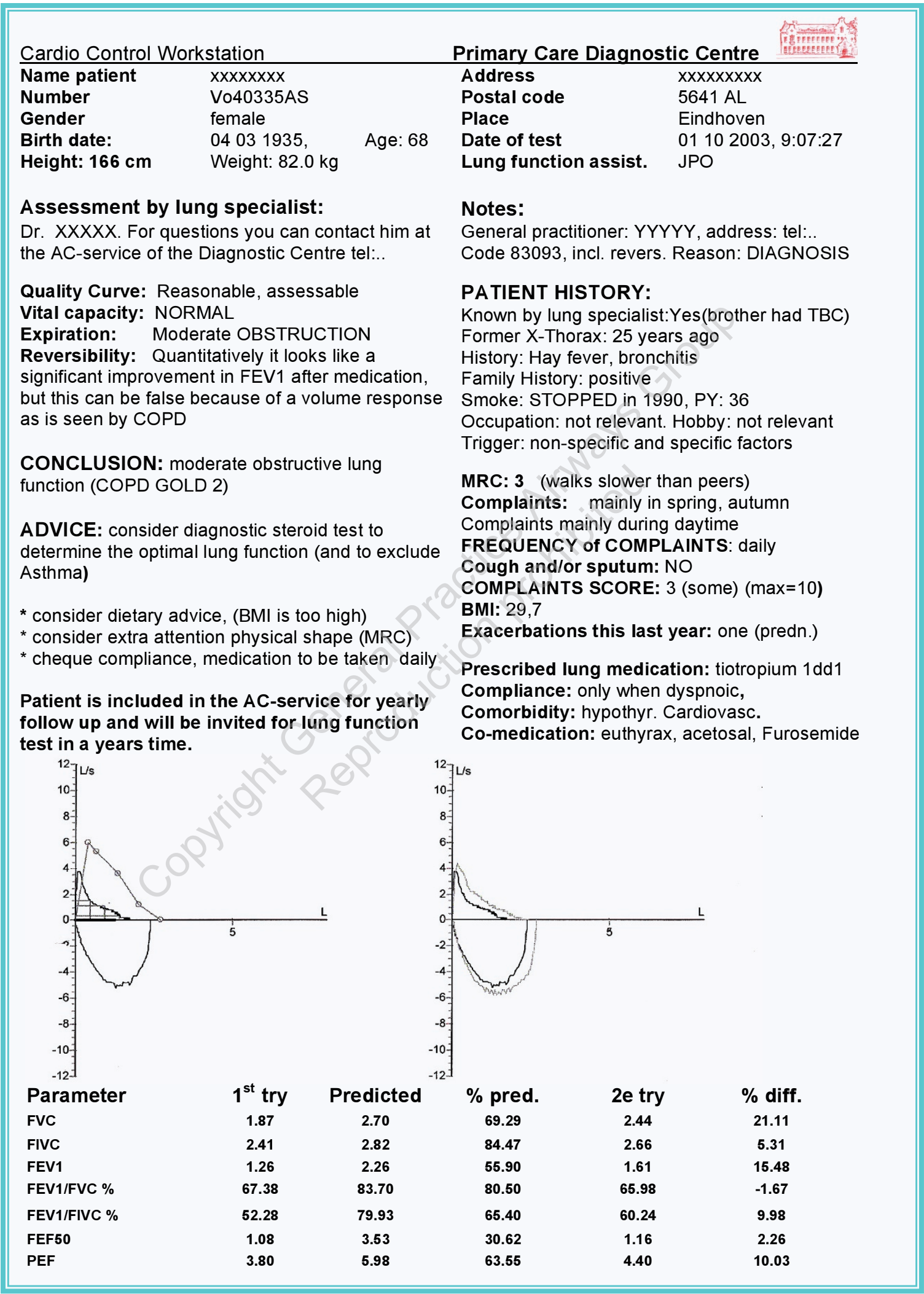


The second research question was addressed by assessing the agreement in the advice for additional diagnostic examinations and the number of patients who were given this advice by each observer. The different types of advice given were:

1. Discrepancy between spirometry results and medical history, search for an alternative diagnosis

2. Normal lung function. When considering asthma perform peak flow registration tests or refer for histamine bronchoprovocation test or repeat spirometry when complaints occur

3. Patient uses inhaled corticosteroids (ICS) but has normal lung function and no complaints. If the diagnosis of asthma has not been confirmed by spirometry consider stopping the use of ICS and repeating the diagnostic spirometry after at least three months

4. Discrepancy between spirometry results and medical history, search for additional diagnoses

5. Spirometry shows obstruction, no reversibility. Perform a steroid test to exclude asthma or to assess the patient's "personal best lung function" (at the time of the study this was according to the Dutch guidelines)

6. Patient uses ICS and has mild obstructive lung function (COPD GOLD 1-2). When there are no frequent exacerbations and no complaints consider stopping the use of ICS to be able to diagnose the actual need for ICS To answer the third question we examined the agreement in assessing the "stability" or "instability" of the clinical condition of the patient. Such reliability of assessment is a prerequisite to agreeing any adjustments in the medical treatment. Since treatment had yet to start in new patients, this part of the assessment only applied to patients who came for follow-up. The options analysed were; "patient condition is stable" or "patient condition is unstable", as per the professional opinion of the respiratory specialists. They took into account the presenting complaints, physical shape, exacerbations, persisting reversibility in asthma patients, and whether or not $\mathrm{FEV}_{1}$ was declining by $>200 \mathrm{ml} /$ year.

\section{Statistical analysis}

Using SPSS 14, $\kappa$ was determined in order to qualify the intraobserver reliability. The inter-observer reliability was expressed as weighted $\kappa .^{16}$

\section{Results}

\section{Patients}

Seventy-two study patients had been referred for diagnostic reasons ("new patients"). Eighty-four patients who were considered to have asthma or COPD by their GP were referred for monitoring ("follow-up patients"). There was no information about whether the diagnoses in this follow-up group were based on spirometry tests or on clinical symptoms. Patients' ages ranged from 18 to 84 years old, and $46 \%$ were men.
According to the pulmonologist reports sent to the GPs, $39 \%$ of the patients had normal lung function with no asthma or COPD, $2 \%$ had a diagnosis of restriction, $23 \%$ of the patients were diagnosed as having asthma, 24\% were diagnosed with COPD, and $12 \%$ had a mixed diagnosis of asthma and COPD.

\section{Quality of spirometry and the reliability of the assessment of reversibility}

Before assessing the reliability of the diagnostic assessment, the quality of the performed spirometry was also assessed. According to ATS criteria $^{17}$ (immediate and rapid incline of the expiration curve, expiration lasting six seconds, no hesitation between expiration and inspiration, and uniting lines of inspiration and expiration) all but 3 of the 156 spirometry tests were of good or sufficient quality. There was almost complete agreement $(\kappa=0.92)$ about this. The agreement in the assessment of reversibility was also good ( $\kappa=0.83$ ).

Reliability of the assessment of the diagnosis asthma and/or COPD

To analyse the intra-observer reliability of the assessed diagnoses, the agreement between the first and the second diagnosis of each patient was analysed for all five assessors. Cohen's kappas for the intra-observer reliability of each assessor were $0.71 / 0.60 / 0.68$ / 0.55 / 0.79, with an average $\kappa$ of 0.67 .

The inter-observer reliability was analysed by comparing the diagnoses of the first assessments between all five assessors. In 114 patients (73\%) there was complete (56\%) to almost complete (17\%) agreement between all five assessors (see Table 1). All types of diagnosis contributed to the 87 fully-agreed assessments (No asthma/COPD - 39; asthma - 18; asthma and COPD - 1; COPD - 29).

Disagreement was likely to be caused by assessment decisions about borderline deviations of lung function from normal: "asthma" or "(about) normal" (21\%), and "COPD" or "(about) normal" (12\%). In $11 \%$ of the patients there was a difference in diagnoses that was difficult to interpret. None of the assessors had a pattern of assessments that was clearly deviant from the others.

Statistically the inter-observer reliability $(\kappa=0.63)$ approximated the reliability found in the intra-observer analysis (see Table 2).

When comparing the diagnostic assessments of new patients and of patients that came for follow-up, a higher intra-observer reliability was found for the new patients. In addition, the inter-observer reliability was better for new patients ( $\kappa=0.69)$ than for follow-up patients $(\kappa=0.48)$

\section{Assessment of the need for additional diagnostic} examinations

An average of $58 \%$ of all patients were given advice to undergo additional diagnostic examinations (to be conducted 
Table 1. Patterns of, and probable cause for, differences between diagnostic assessments by five respiratory specialists of 156 primary care patients based on spirometry data and medical histories.

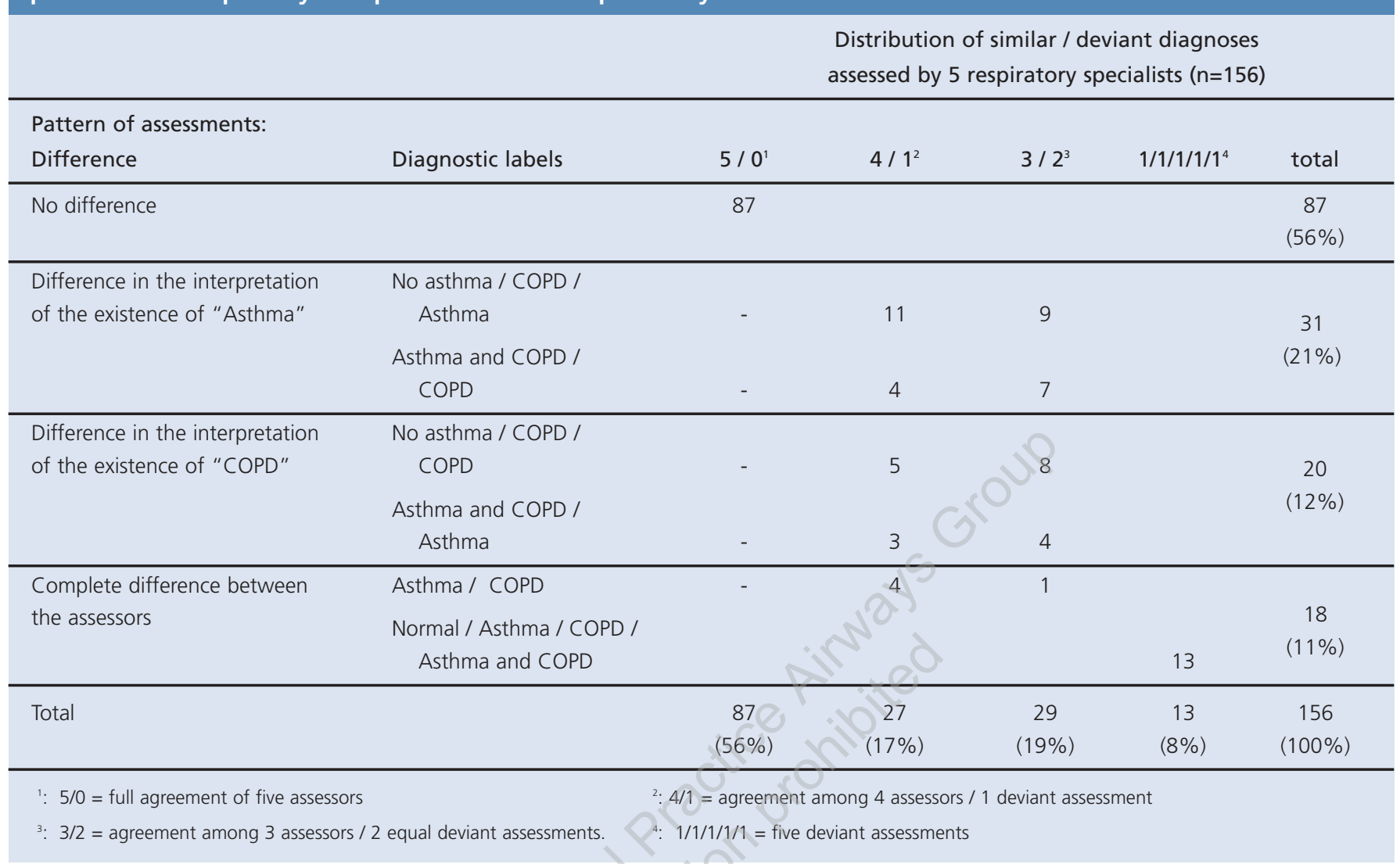

Table 2. Intra- and inter-observer reliability in diagnostic assessments performed by respiratory specialists and based on written information about lung functions and medical histories.

\begin{tabular}{lccc}
$\begin{array}{l}\text { Agreement in diagnoses } \\
\text { (No Asthma; Asthma; Asthma and COPD; COPD) }\end{array}$ & $\begin{array}{c}\text { New patients } \\
\kappa\end{array}$ & $\begin{array}{c}\text { Follow-up patients } \\
\kappa\end{array}$ & $\begin{array}{c}0,56 \\
\kappa\end{array}$ \\
\hline $\begin{array}{l}\text { a. Intra-observer reliability } \\
\quad \text { (Mean } \kappa \text { and range) }\end{array}$ & $\begin{array}{c}0,66 \\
(0,55-0,74)\end{array}$ & $(0,40-0,79)$ & $(0.55-0,79)$ \\
\hline b. $\begin{array}{l}\text { Inter-observer reliability } \\
\text { (Weighted } \kappa \text { ) }\end{array}$ & 0,69 & 0,48 & 0,63
\end{tabular}

by their GPs) after the first assessments and $68 \%$ after the second. The number of patients recommended for referral to a specialist because of severe problems (lung function decline and/or symptoms) was 8\% (10-25\%) in the first and 7\% (5$19 \%$ ) in the second series of assessments.

The intra-observer reliability for advising additional diagnostic examinations had a reasonable average Cohen's kappa of 0.50 but there were big differences between assessors, as Cohen's $\kappa$ ranged from 0.31-0.62. The advice for new patients showed better reliability $(\kappa=0.54-0.58)$ than the advice for follow-up patients $(\kappa=0.38-0.40)$, but did not meet our criteria for assessing a weighted $\kappa$ (Table 3 ).

\section{Assessment of the clinical condition of the follow-up patient}

Agreement in assessing the clinical stability of the follow-up patients scored low for intra-observer reliability: Cohen's kappa for each assessor was 0.64 / 0.39 / 0.35 / 0,24 / 0.28, (mean $\kappa:$ 0.38). Based on this finding the inter-observer weighted kappa was judged to be of no value and was not analysed any further.

\section{Discussion}

The main finding of our study is that there was moderate agreement (inter-observer reliability) and consistency (intra- 
AEM Lucas et al.

Table 2. Intra-observer reliability of the advice for additional diagnostic examinations given in case of uncertainty of the diagnosis based on spirometry and written patient history data.

Advice for additional diagnostic examination

Agreement between repeated assessments for each assessor mean $\kappa$ (range)

1. Discrepancy between complaints and spirometry

$\rightarrow$ check for other diagnosis

$0,54(0,32-0,65)$

2. Obstruction, no diagnosis yet,

$\rightarrow$ perform steroid test

$0,58(0,40-0,86)$

3. Normal spirometry, possibly asthma

$\rightarrow$ peakflow-registration test or histamine bronchoprovocation test

$0,56(0,24-0,75)$

4. Discrepancy between complaints and diagnosis

$\rightarrow$ check for additional medical problem

$0,38(0,03-0,67)$

5. Use of ICS for unclear reason

$\rightarrow$ stop ICS, repeat FVM after three months

$0,40(0,13-0,60)$

Advice $\mathrm{nr} 1,2$ and 3 can be given to new patients, advice $\mathrm{nr} 4$ and 5 to follow-up patients.

observer reliability) in the diagnostic assessments of asthma and COPD as performed by different assessors based on spirometry and written information about a patient's history, complaints and compliance. We could not draw conclusions about the reliability of the advice for additional diagnostic examinations. The assessment of patients' clinical stability was found to be statistically not reliable; therefore, this therapeutic advice should be treated with caution by the GP. Comparable diagnostic assessments of asthma/COPD Intra- and inter-observer reliability in the diagnostic assessment of asthma/COPD is hardly ever discussed in the literature. A previous analysis of variability in the interpretation of spirometry tests by respiratory specialists showed $63 \%$ agreement. ${ }^{18}$ In another study, interns and respiratory specialists reached $58 \%$ agreement. ${ }^{19}$ The concordance we found in this study (mean $\kappa=0.64$ ) was comparable - which is remarkable since the respiratory specialists combined the interpretation of medical history and spirometry data thus making the assessment more complicated. When compared to other complicated diagnostic procedures such as chest radiograph interpretation by pulmonologists $(\kappa=0.51),{ }^{20}$ the diagnostic reliability of the assessments in our study is not inadequate.

\section{What is the maximum achievable reliability?}

This finding of moderately good agreement was surprising, for one would expect that diagnostic concordance would be high. To understand this better we examined in detail the discrepancies between the diagnoses assessed by the pulmonologist who had the best intra-observer reliability $(k=0.79)$. In 25 out of 156 cases $(16 \%)$ different decisions were made. Twelve cases concerned differences in diagnosing patients with lung function that was borderline to normal (FEV 1 around $80 \%, \mathrm{FEV}_{1} / \mathrm{FVC}$ around 0.68 ). Other doubts were caused by the interpretation of an improvement in $\mathrm{FEV}_{1}$ as a volume response or as 'real' reversible airway obstruction that can be seen in asthmatics $(\mathrm{N}=5)$ and by discrepancies between complaints and spirometry results $(\mathrm{N}=3)$. The use of ICS without an obvious indication caused different diagnoses in five out of 36 of those cases.

It is unlikely that these "real life problems" can be completely solved by a strict assessment protocol. This might indicate that expectations about high concordance for diagnostic support services or outpatient clinics are not realistic.

An interesting question is whether GPs themselves, willing to overcome the practical problems of performing spirometry, do better. Trained GPs have been shown to identify normal and clearly deviant spirometry. ${ }^{21}$ They also had problems in interpreting the minor variations. Further research could show how GPs - be they well trained or not - diagnose the combination of spirometry and medical history and how this compares to the paper assessments of the respiratory specialists of the asthma/COPD-service.

\section{Assessments in new and follow-up patients}

The reliability of the diagnostic assessments was better in new patients than in patients who came for follow-up. This can be explained by the following problem we encountered: several patients referred as new patients used ICS without a diagnosis of asthma confirmed by former spirometry. In fact, because of their use of ICS, they had to be considered as follow-up patients. When their use of ICS could not be explained by the results of the actual spirometry test or the 
patient's medical history, the assessing pulmonologist had to choose: either he accepted the presumed diagnosis (asthma or moderate COPD with recurrent exacerbations), or he refused to do so and postpone the diagnosis until the use of ICS was stopped and spirometry was renewed. As we found in another study, this dilemma exists in about $20 \%$ of all patients referred to the diagnostic service 22 and the possible conflict in decision-making can influence (negatively) the reliability of the diagnostic assessment in follow-up patients.

Assessment of the need for additional diagnostic examinations

The value of additional diagnostic advice was shown in a former study where about $50 \%$ of the patients were given advice which led to a large number of other or additional diagnoses (rhinitis, gastric asthma, cardiac problems, etc). ${ }^{11}$ Also, more than $50 \%$ of all patients we describe in this paper received advice regarding additional examinations. The most important effect this advice might have had is that it encouraged the GPs to explore differential diagnostic considerations in case there was a discrepancy between spirometry findings and the patient's symptoms.

\section{Assessment of the patients' clinical condition and medical advice}

To classify the stability of a patient's disease one should take into account their complaints/symptoms, physical shape, exacerbations, use of reliever medication, etc. ${ }^{23}$ In this study we found that the clinical stability of a patient could not be assessed reliably from the written information. Consequently, the (pharmacotherapeutical) treatment adjustments showed a great intra- and inter-observer variability. To improve this, a better algorithm will be needed for assessing clinical stability. But even when there is such a protocol, the asthma/COPDservice will only be supportive to the GP in identifying instability and will not discharge the GP from taking final responsibility and consequently weighing up the therapeutical advice offered.

\section{Difficulties encountered in the study}

In this paper we studied the regular assessment procedure of the asthma/COPD-service without making corrections for patients who were referred with a wrong indication (referred for follow-up without a diagnosis). In addition, no special instructions were given to the respiratory specialists about how to handle specific situations such as the use of ICS by patients without obvious reasons for that use. Therefore, our results reflect the "every day life" reliability of the assessments of the asthma/COPD-service to a greater extent than the reliability of the professional assessment skills of the pulmonologists.

Our study identified the need for a properly-assessed diagnosis (before medical treatment) in order to monitor reliably an asthma or COPD patient. As a result of our study the asthma/COPD-service developed referral instructions for the GPs. In addition, regular interviewing between the respiratory specialists will be organised to develop and discuss extra guidelines for the assessments. When these changes have been implemented we intend to do a follow-up study examining the expected improvements in the reliability of the diagnostic and therapeutic assessments.

\section{Lessons for clinical practice as a result of the study}

Not all GPs have the facilities, the skills or the wherewithal to perform adequately the diagnostic procedures necessary to identify correctly asthma or COPD (or its absence). GPs experience the need for ongoing support with regard to the management of the care process ${ }^{24}$ and spirometry interpretation. ${ }^{25}$ Services offering such support need to validate their procedures and need to provide the best reliable reports to the GPs using that service. The reliability of the advice varies. GPs referring to the service should realise that $100 \%$ reliability cannot be expected because of the complexity of diagnostic procedures.

\section{Funding}

The author (AEML) is supported by an unrestricted grant from "Picasso," an initiative of the research institution Caphri, Pfizer Inc. and Boehringer Ingelheim Inc.

\section{Ethics approval}

Not applicable

\section{Competing interests \\ None}

\section{Acknowledgements}

We gratefully acknowledge the commitment of the participating pulmonologists from the Eindhoven Catharina Hospital; and the lung function assistants, secretary and staff of the Asthma/COPD-service in administering the study.

\section{References}

1. Walters JA, Mudge P, Johns DP, Walters EH, Wood-Baker R. Barriers to the use of spirometry in general practice. Aust Fam Physician 2005;34(3):2001-03.

2. Frank TL, Hazell ML, Linehan MF, Frank PI. The diagnostic accuracies of chronic obstructive pulmonary disease (COPD) in general practice: the results of the MAGIC (Manchester Airways Group Identifying COPD) stud. Prim Care Resp J 2006;15(5):286-93. http://dx.doi.org/10.1016/j.pcrj.2006.07.007

3. Bolton $\mathrm{CE}$, lonescu $\mathrm{AA}$, Edwards $\mathrm{PH}$ et al. Attaining a correct diagnosis of COPD in general practice. Respir Med 2005;99(4):493-500. http://dx.doi.org/10.1016/j.rmed.2004.09.015

4. Jones R, Whittaker M, Hanney K, Shackell B. A pilot study of a mobile spirometry service in primary care. Prim Care Resp J 2005;14(3):169-71. http://dx.doi.org/ 10.1016/j.pcrj.2004.12.003

5. Walker P, Mitchell P, Diamantea F, Warburton CJ, Davies L. Effect of primarycare spirometry on the diagnosis and management of COPD. Eur Respir J 2006; 28(5):945-52. http://dx.doi.org/10.1183/09031936.06.00019306

6. White P W, Wong T, Fleming B. Gray. Primary Care spirometry: test quality and feasibility and usefulness of specialist reporting. Br J Gen Pract 2007;57(542): 701-5.

7. Hassett R, Meade K, Partridge MR. Enhancing the accuracy of respiratory diagnoses in primary care: a report on the establishment of a Community Respiratory Assessment Unit. Prim Care Resp J 2006;15(6):354-61. http://dx.doi.org/10.1016/j.pcrj.2006.10.003 
AEM Lucas et al.

8. Vaughn R, Carter R, Maclntyre D. An outreach Spirometry service for Greater Glasgow health board: does it help in diagnosis. Eur Respir J 2004;24(suppl. 48):182s.

9. Wolfenden $H$, Bailey L, Murphy K, Partridge MR. Use of an open access spirometry service by general practitioners. Prim Care Resp J 2006;15(4):252-5. http://dx.doi.org/10.1016/j.pcrj.2006.05.007

10. Denis JPC, van Hessen PAW. Asthma/COPD service improves diagnosis and monitoring in primary care. Eur Respir J 2005;26(suppl.49):178s.

11. Kuilboer MM, van Wijk MA, Mosseveld M, et al. Feasibility of Asthma Critic, a decision-support system for asthma and COPD which generates patientspecific feedback on routinely recorded data in general practice. Fam Pract 2002;19(5):442-7. http://dx.doi.org/10.1093/fampra/19.5.442

12. Lucas A, Smeenk F, Smeele I, Brouwer T, van Schayck O. Validity of diagnostic support of an asthma/COPD-service in primary care. $\mathrm{Br} J$ Gen Pract 2007;57(544):892-6. http://dx.doi.org/10.3399/096016407782317883

13. Pauwels RA, Buist AS, Calverley PM, Jenkins CR, Hurd SS. Global strategy for the diagnosis, management and prevention of chronic obstructive pulmonary disease. NHLBIMHO Global Initiative for Chronic Obstructive Lung Disease (GOLD). update 2005. Am J Respir Crit Care Med 2001;163(5):1256-76.

14. Geijer RMM, Thiadens HA, Smeele IJM et al. Standard of he Dutch College of General practitioners on asthma and COPD; diagnostics (in Dutch). Huisarts Wet 2001;44(3):107-17.

15. Geijer RMM, Thiadens HA, Smeele IJM, et al. Standard of the Dutch College of General practitioners on asthma and COPD: treatment (in Dutch). Huisarts Wet 1997;44(5):2007-219.

16. Leiss.exe V1.0 - Calcul du coefficient Kappa multi-juges d'après Fleiss ; Auteur: Philippe Bonnardel* http://perso.worldonline.fr/kappa/
17. Miller MR, Hankinson J, Brusasco V, et al. Standardisation of spirometry. Eur Respir J 2005;26:319-38. http://dx.doi.org/10.1183/09031936.05.00034805

18. Quadrelli SA, Roncoroni AJ, Porcel G. Analysis of variability in interpretation of spirometric tests. Respiration 1996;63(3):131-6. http://dx.doi.org/ $10.1159 / 000196532$

19. Hnatiuk O, Moores L, Loughney T, Torrington K. Evaluation of internists' spirometric interpretations. J Gen Intern Med 1996;11(4):204-08. http://dx.doi.org/10.1007/BF02642476

20. Bolton JS, Padia SA, Borja MC, et al. The predictive value and inter-observer variability of donor chest radiograph interpretation in lung transplantation. Eur J Cardiothorac Surg 2003;23(4):484-7. http://dx.doi.org/10.1016/S10107940(03)00016-2

21. Chavannes N, Schermer T, Akkermans R, et al. Impact of spirometry on GP's diagnostic differentiation and decision-making. Respir Med 2004;98(11):112430. http://dx.doi.org/10.1016/j.rmed.2004.04.004

22. Lucas AEM, Smeenk F, Smeele IJM, van Schayck CP. Overtreatment with inhaled corticosteroids and diagnostic problems in primary care patients, an explorative study. Fam Pract 2008;25(2):86-91. http://dx.doi.org/10.1093/fampra/cmn006

23. Jones PW, Agusti AG. Outcomes and markers in the assessment of chronic obstructive pulmonary disease. Eur Respir J 2006;27(4):822-32.

24. Meulepas MA, Jacobs JE, Lucas AE, et al. The feasibility of a primary care model for the management of COPD. Prim Care Resp J 2006;15(6):337-41. http://dx.doi.org/10.1016/j.pcrj.2006.08.010

25. Poels PJ, Schermer TR, Akkermans RP, et al. General practitioners' need for ongoing support for the interpretation of spirometry tests. Eur Respir J 2007; 13(1):16-19.

\section{Available online at http://www.thepcrj.org}

\section{CORRIGENDUM}

\section{Corrigendum to 'Asthma Programme in Finland: the quality of primary care spirometry is good' (Prim Care Resp J 2008;17(4):226-231)}

\section{*Leena E Tuomisto a, Vesa Jarvinen ${ }^{b}, J^{\prime}$ ukka Laitinen`, Marina Erholad, Minna Kaila ${ }^{\mathrm{e}}$, Pirkko E Brander ${ }^{f}$}

The authors regreat that an error occurred on page 227, Figure 2

The first box in the flow chart reads 'Referral letters to three pulmonary units during year $2001^{11}$ '

should read

'Referral letters to three pulmonary units during year $2001^{14 \prime}$

\footnotetext{
*Corresponding author: Dr Leena Tuomisto, Pulmonary Department, Seinajoki Central Hospital, Huhtalantie 53, Seinajoki, 60220, Finland Tel: +358408457163 Fax:+35864154989 E-mail: leena.tuomisto@gmail.com
}

DOI of original article: doi:10.3132/pcrj.2008.00053 